\title{
A Composite Approach to Ives's 'Cage'
}

\section{SCOTT MURPHY}

\begin{abstract}
Larry Starr's analysis of Charles lves's 'The Cage', in his book A Union of Diversities, contrasts with most readings of the song in that he focuses on how the points of congruence between the highly dissimilar vocal and piano parts form a 'powerful unity'. However, he provides no specific analytical method for examining this unity more closely. This study offers one such method for 'The Cage', revealing a path within a virtual, bounded space that correlates with some of the syntactic and semantic aspects of the song's text. These syntactic correlations also hold true in a similar passage in lves's song 'Majority'.
\end{abstract}

Charles Ives's song 'The Cage', presented as Example 1, provides students of twentiethcentury music with concise examples of whole-tone collections and quartal harmonies, neatly isolated in the vocal part and the piano part respectively. ${ }^{1}$ Since these two dissimilar parts occur simultaneously, the song also illustrates Ives's well-documented penchant for juxtaposing contrasting ideas. Many interpretations of this song understandably embrace and encourage this segregation, either by emphasizing the differences between the two parts, or by channelling analytical energies to each part separately. In contrast, Larry Starr's reading of the song suggests an analytical benefit from listening to the interaction between these two parts. ${ }^{2}$ However, he provides no specific analytical method for examining this interaction, or at least no method that approaches the sophistication of the methods that have been previously applied to each individual part. This study offers one such method for 'The Cage', revealing a path within a virtual, bounded space that correlates with some of the syntactic and semantic aspects of the song's text. These syntactic correlations also hold true in a similar passage in Ives's song 'Majority'.

A version of this essay was presented at the 2003 Conference on Music and Gesture in Norwich, England. I would like to thank Larry Starr, Philip Lambert, and two anonymous readers for reading and commenting upon an earlier draft of this article.

1 'The Cage' first publicly appeared as no. 64 in the composer's 114 Songs, published in 1922 . However, the song is based upon an earlier chamber work entitled 'In the Cage', which was sketched in 1906 and eventually published in 1932 as the first movement of A Set of Pieces for Theater or Chamber Orchestra. A new version of 'The Cage', as no. 57 in the late H. Wiley Hitchcock's recent edition of Ives's 129 Songs, takes into account these three sources, among others. The score in Example 1 generally follows Hitchcock's version, reflecting his choice to write chord 19 with a $\mathrm{D}^{3}$ and $\mathrm{F}^{3}$, as in the 1906 score-sketch and the 1932 chamber work, instead of with a $\mathrm{D}^{3}$ and $\mathrm{F}^{3}$ as in the original 1922 publication of the song. Hitchcock discusses some of the issues raised in the creation of this new edition in 'Editing Ives's 129 Songs'; pages 65-70 focus on 'The Cage'. (In an apparent oversight, all the sharps are missing in the fifth chord of the first bar in Hitchcock's edition; this has been rectified in Example 1.)

2 Starr, A Union of Diversities, 126-9. 

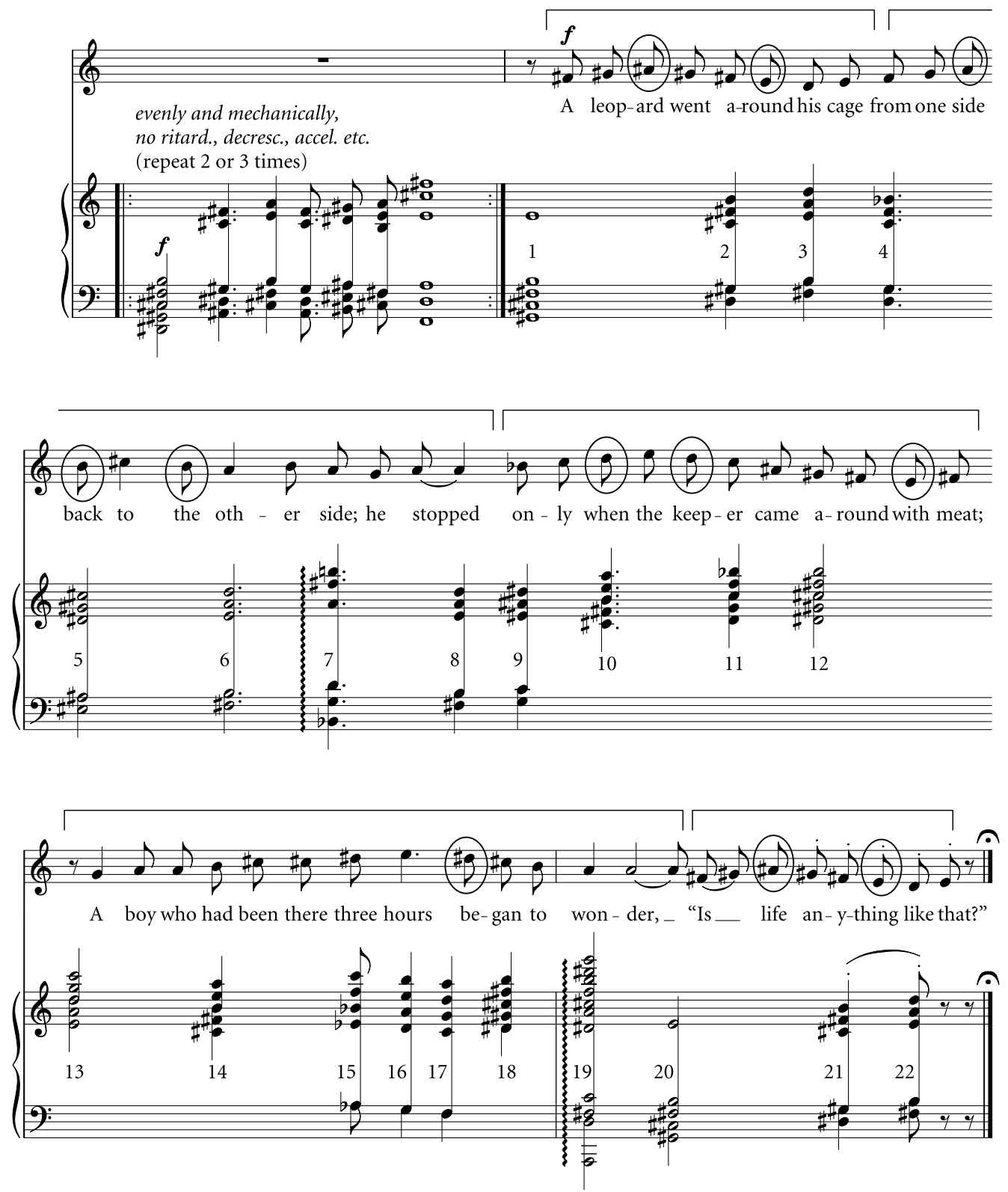

Example 1 Charles E. Ives, 'The Cage'. ( ) 1955, Peer International Corporation. International Copyright Secured. Reproduced by permission of Faber Music Limited, London. Numerical labelling of chords and brackets above vocal line follow Lambert, 'Ives and Berg', 116, and The Music of Charles Ives, 152.

Encircled vocal notes are not doubled in the piano chord that accompanies them. Accidentals apply only to notes that they immediately precede.

Daniel Kingman's one-sentence summary of 'The Cage' is a concise example of how the two parts of the song are customarily disconnected from one another: 'The voice intones Ives's short prose text, again using mostly the noncommittal whole-tone scale, while the piano, in a rhythmically independent part, uses severe sonorities based on the interval of the 
perfect fourth, to depict the restless pacing of the leopard in the cage. ${ }^{3}$ Cassandra Carr's reading, as part of a longer article-length study on the composer's humour as demonstrated in his songs, applies to this contrast a more subjective interpretation. After discussing the whole-tone vagaries of the vocal line, she continues:

Further intensifying the unsettled quality of the song is the refusal of the voice line and the accompaniment to mesh [emphasis mine]. Indeed, the accompaniment chords are quartal in nature, insuring a continued sense of displacement irrespective of the shifts and turns of the vocal line. Thus 'The Cage' succeeds in evoking a sense of wry philosophical humor far more lasting than its own brief span. ${ }^{4}$

Philip Lambert, in an article comparing Ives's and Berg's compositional practices, separates the vocal line and piano part of 'The Cage' to exemplify two different uses of cyclically generated pitch-class sets. ${ }^{5}$ Lambert finds an underlying logic for the structure of each part. In expressing five-note and six-note whole-tone collections, the vocal line switches from one whole-tone collection to the other four times, creating a five-part structure to the melody, as shown with the brackets in Example 1. (The fourth section includes one non-collectional pitch: the E on 'hours'.) The end of each of these five parts coincides with the end of a textual phrase except in the case of the word 'stopped', where a caesura makes sense for semantic reasons. In the piano part Lambert correlates the perfectfourth registral transposition of the opening bar that produces chords 1-7 with the generating interval of the quartal sonorities themselves, and discovers that chords $8,10,13$, 15 , and 18 are, in order, related by $\mathrm{T}_{3}$ to the first five chords of the opening bar. The one relationship Lambert sees between the quartal piano part and the whole-tone vocal part (besides the fact that the sets for both are cyclically generated) is found at the point where the piano line most deviates from quartal harmony: chord 19 presents a complete wholetone collection in the treble staff.

In his dissertation, Shaugn O'Donnell concentrates almost exclusively on the piano part of 'The Cage', creating Klumpenhouwer-style networks whose nodes are either pitches of a piano chord, whole piano chords, or sequences of piano chords. ${ }^{6}$ The similarities (or isographies, as they are called in Klumpenhouwer-network theory) among these networks reveal various degrees of correspondence among hierarchical levels. Only one of his seventeen networks comprises the pitches from the vocal line-specifically, the lowest pitch in each of the five sections of the vocal line bracketed in Example 1-and is found to be weakly related (by means of what David Lewin dubbed negative isography) to three piano networks. However, this isography does not participate in the higher-level connections O'Donnell proposes, which illustrate a unity among the five formal sections of the song.

3 Kingman, American Music: a Panorama, 462.

4 Carr, 'Charles Ives's Humor as Reflected in His Songs', 135.

5 Lambert, 'Ives and Berg', 115 (vocal line) and 118 (piano part).

6 O’Donnell, 'Transformational Voice Leading in Atonal Music', 114-26. Klumpenhouwer networks were first presented in Klumpenhouwer, 'A Generalized Model for Voice-Leading in Atonal Music', and the term 'isography' and its modifiers 'strong', 'positive', and 'negative' are defined in Lewin, 'Klumpenhouwer Networks', 83-120. 
Against this backdrop, Larry Starr's treatment of the song towards the end of his book $A$ Union of Diversities: Style in the Music of Charles Ives stands out as an exception. Most of Starr's analyses follow from the basic premise that 'when [the juxtaposition of different styles] takes place, we can no longer speak of the music as being composed in a particular style. Rather, the music is composed with styles; the whole issue of style becomes part of the music's subject matter, on a very profound level. ${ }^{37}$ Therefore Starr's interpretations concentrate on Ives's stylistic heterogeneity, rather than using traditional analytical methods that 'seek to find unity and coherence despite the variegated surface'. ${ }^{8} \mathrm{He}$ finds logical orderings in Ives's arrangements of successive styles in his music; for example, he finds a stylistic arch form in the songs 'Ann Street' and 'Walking', as well as in the chamber piece Scherzo: Over the Pavements.

Turning his attention away from compositions that present a succession of styles towards those that present a simultaneity of styles - what he calls 'layering' - Starr notes two varieties of this practice: 'One is the creation of new composite styles from conceptually separable elements [...]. In such cases, the resulting music is experienced by the listener in terms of a single, albeit complex, stylistic unit. In the other form of layering, the emphasis is on multiplicity rather than combination, as the listener experiences the coexistence of two or more independent stylistic planes. ${ }^{9}$ Starr clearly places 'The Cage' in the former category; moreover, it is his only 'instructive example of [Ives's] use of layered elements to construct a single composite style'. ${ }^{10}$ He begins his analysis as Kingman and Carr might, by identifying the differences between the pitch structures of the piano and vocal parts, and recognizing that 'since a whole-tone scale contains no perfect fourths, it is apparent that the voice and piano parts are constructed along different lines'. ${ }^{11}$ But the rest of the four-paragraph analysis makes a case that this song 'offers two distinctive kinds of musical vocabulary inextricably bound together in a powerful, if artificially imposed, unity. ${ }^{12}$ He supports this view by pointing out intersections between the pitch and rhythmic content of the piano and vocal parts.

Although the song has no traditional sense of meter, the voice and the accompaniment maintain a feeling of rhythmic alignment through the song. 'The Cage' never approaches the feeling of two completely independent rhythmic layers [compare to Kingman's statement above] [...]. Perhaps more significantly, the many points of exact rhythmic alignment in 'The Cage' - when the voice and the piano attack notes together - are almost always points of pitch alignment as well, where the vocal pitch will also be found within the piano's chord. (In the two cases that constitute the only exceptions to this, on the words 'back' and 'when', the following vocal pitch is a

7 Starr, A Union of Diversities, 9.

8 Starr, A Union of Diversities, 26.

9 Starr, A Union of Diversities, 115.

10 Starr, A Union of Diversities, 126.

11 Starr, A Union of Diversities, 128.

12 Starr, A Union of Diversities, 128-9. 
member of the piano's chord.) This pitch alignment helps the singer, of course, but in doing so assures a real and continuing sense of ensemble between the performers that cannot help but communicate itself to the listener as well [...]. The music of 'The Cage' is constructed so as to emphasize continuously the points of convergence between its two different pitch systems. ${ }^{13}$

Lambert's book on Ives reserves a chapter for 'The Cage', and, although most of the chapter treats the piano and vocal parts separately, incorporating the observations from his Ives/Berg article as well as some new ideas, he too acknowledges this pitch overlap, specifically as a manifestation of the close relationship between $\mathrm{T}_{5}$ and $\mathrm{T}_{2}$ cycles. 'In all, only chord 17 does not harmonize any of the notes it accompanies; all the other chords include at least one, and often all of the concurrent notes in the melody. ${ }^{14}$ He too recognizes that the non-quartal piano chords 7 and 19 also contain a synchronous vocal pitch A.

Although these observations of an overlap between piano and vocal parts are significant, in that they help to complement interpretations that treat the two parts independently, is there anything worth noticing beyond the recognition that the two parts overlap? Or, to put it more precisely, when Starr claims that the pitch alignment between the vocal and piano parts 'communicates itself to the listener', is this alignment the message or the medium of this communication? One may be content to hear it as the message itself: the fact that the piano and vocal parts, in spite of their different harmonic constructions and often divergent trajectories, still manage to connect frequently in pitch-class space comes across as akin to a bit of choreographic virtuosity. But as a medium for information, the binary state of overlap/non-overlap is inherently limited in what it can communicate: either a vocal pitch class is doubled in its accompanying piano chord, or it is not. Example 1 differentiates these two types of vocal notes by means of circles around the non-doubled pitches. This differentiation reveals some curious things, such as how the notes for 'back to the' and 'when the keep-' invert the customary roles of chord notes and non-chord notes in a neighbour-note pattern, which is rectified in the notes for 'round with meat'. However, another way to make some sense of this 'single composite style' is to refine the meaning of overlap here, in the hope of creating a richer information base for a new analytical perspective.

Consider the piano chords 8 and 9 and the vocal notes $\mathrm{A}^{4}$ and $\mathrm{B} b^{4}$ that they accompany. Each pitch is doubled by the concurrent piano harmony, creating overlap between the pitch collections of the two parts. (Hereafter, vocal notes that are so doubled will be designated as chord notes, even though common practice often demonstrates that a melodic pitch does not have to be doubled in the accompaniment to be considered a chord note.) But these two chord notes have more than that in common: the two vocal notes are at the same 'height' within their respective chords, in that each is one perfect fourth from the top of its accompanying quartal chord. One could say simply that piano and voice are moving in parallel motion here, since both parts are transposed up a semitone. But, by concentrating

13 Starr, A Union of Diversities, 128.

14 Lambert, The Music of Charles Ives, 157. 
less on absolute registral space and more on the contextual space defined by the structure of the harmonies, one may record no 'motion' at all, in that the vocal pitches have remained at the same 'height' relative to their supporting harmonies. Or, in other words, the net difference between the motion of the vocal line and the piano part is zero. ${ }^{15}$ Following Starr's choice of words, collaborative motions such as these and any analysis or representation that incorporates or expresses such collaborative motions will be referred to as composite.

This composite stasis continues into chord 10 and the voice's chord note $\mathrm{E}^{5}$ : once again the melodic note is one perfect fourth from the top of the chord, so, relative to the quartal structure of the harmony, these three melodic chord notes still have not travelled anywhere. This is the only time in the song when the composite motion is zero; appropriately, this stasis immediately follows the text's only reference to a cessation of activity. Certainly other aspects of the music symbolize the word 'stopped' - notably the longest duration so far in the vocal line - but a composite reading conveys the ensuing impression of staying put, a customary dining behaviour for cats of prey. Also notice how this frees the narrator from the literal text-painting of 'stopped', allowing the text and the story to continue, while communicating a lingering representation of the word.

An analogy with relative motion inside and outside Einstein's boxcars fits well here. ${ }^{16} \mathrm{~A}$ listener who focuses on the individual trajectories of the piano and vocal parts through registral space and/or pitch-class space is analogous to a stationary observer outside a moving boxcar carrying a passenger. In the case of the excerpt discussed above, the passenger (the voice's chord notes $\mathrm{A}^{4}, \mathrm{~B} b^{4}$, and $\mathrm{E}^{5}$ ) is seated in the boxcar (piano chords 8-10). To an outside observer, it appears that both boxcar and passenger are moving. However, an observer on the inside of the boxcar sees the passenger as stationary: the passenger has not changed her location relative to the boxcar. To interpret the leopard's motion as 'stopped' during these three chords therefore requires the listener to come inside the boxcar - or to use the analogous space from the text, to come inside the cage - and to hear the motion of the voice-as-leopard relative to this confined space. ${ }^{17}$

15 At one point in A Union of Diversities, Starr asks: 'Can there be art which makes stylistic divergence its subject matter - and, even more than that, its message and meaning?' (10). In the case of composite analysis, the net difference of the transpositions of two different layers through the same pitch or pitch-class space may be understood as one quantification of this 'divergence'.

16 One of Einstein's famous 'thought experiments' that led to the theory of special relativity involves a boxcar moving forward at tremendous speed with observers both inside and outside the boxcar. When a lightbulb is turned on in the middle of the boxcar, the observer inside the boxcar sees the light strike the front and back walls of the boxcar at the same time. However, an observer outside the boxcar sees the light strike the back wall first, and then strike the front wall second. This experiment demonstrates how maintaining the same speed of light for all observers can result in different perceptions of the same phenomena.

17 Among the many interpreters of 'The Cage' who associate musical components of Ives's song with the leopard and/or the cage (Ursula Henrietta Euteneuer-Rohrer, Cassandra Carr, Daniel Kingman, Philip Lambert, David Nicholls, and Larry Starr), only Starr, upon whose notion of a 'composite style' my analytical approach to 'The Cage' is based, associates the vocal line with the leopard and the piano part with the cage: 'The vocal line [...] circles back and forth aimlessly, like the leopard it is describing; it seemingly 'bumps' at intervals against the leaden piano chords, which may well be taken to suggest something like the bars of a cage. (These chords even look something like cage bars in Ives's score.)' (A Union of Diversities, 128) 
The first appearance of the vocal part projects a different kind of composite motion. The opening $F \sharp$, whether sung in the male or female range, finds a pitch-class match with the $\mathrm{F}^{3}$, which lies right in the 'middle' of chord 1: two perfect fourths from both the top and bottom of the chord. When the F\# returns again on the first syllable of 'around' above chord 2, the vocal line may have returned to the same spot, but the harmonic shift reorients the position of this vocal note relative to the harmony: it now doubles the harmonic note one perfect fourth from the top. Then the voice's low D on the word 'his' matches the top note in chord 3. Therefore, the composite motion for these three melodic-harmonic pitch-class unions is linear through the quartal structure: from the centre to the upper limit via two ascending perfect fourths. As before, this linear motion may be understood as the net differences between the piano and vocal parts. From chord 1 to chord 2, the opening F\#s stay the same (0) and the piano part ascends a perfect fifth (7): therefore the net difference of pitch-class motion is $0-7=5$ (pitch-class interval 5: up a perfect fourth or down a perfect fifth). Had the net difference been 0 - in other words, had the voice moved in parallel with the piano, as in the motion between chords 8 and 9 described above - the vocal pitch class above chord 2 would have been $\mathrm{C} \#$. The net difference of 5 here represents the extent of the voice's deviation from parallel motion: the vocal note is notionally 'displaced' from $\mathrm{C} \#$ to $\mathrm{F} \#$ by pitch-class interval 5. From chord 2 to chord 3, the vocal line drops a major third ( 8 in pitch-class interval terms) and the harmony rises a minor third (3): $8-3=5$. Associating this motion with the leopard once again, it portrays a more deliberate action - moving from the centre of the cage to one of the sides - than the nervous undulations of the vocal line alone.

Ives's particular choice of the quartal pentachord as the primary harmony permits its spatial correlation with a cage in two ways. First of all, the piano chords are formed from adjacent members of an interval cycle, allowing the generating interval to serve as a consistent unit of measure. This unit will be referred to as a step; however, it should not be confused with conventional notions of 'stepwise voice leading'. In conventional terms, the $\mathrm{A}$ and $\mathrm{B} b / \mathrm{A} \#$ in chords 8 and 9 respectively are separated by a step in the vocal line. But in terms of composite motion, there is no step between the two notes, as the A in chord 8 occupies the same position in the chord (one perfect fourth from the top) as the $\mathrm{B} b$ in chord 9. By contrast, the F\# in chord 1 moves one step to the F\# in chord 2, since, from the one vertical to the next, it moves one position up within the chord. Also, the fact that the harmonies do not express a complete interval cycle allows a correlation with a spatial dimension such as 'high to low', or 'left to right', even within the domain of pitch class. Were the chords fully-diminished seventh chords or whole-tone hexachords, this would not be possible, for there would be no boundaries to the set that would confer a total ordering upon its constituents and represent the cage's confines. Such finite, totally ordered sets will be referred to as segments, and the two extremities of any segment are its limits. As demonstrated in the two preceding analyses, each of the voice's chord notes may be assigned relative ordinal positions within the accompanying piano segments - as $n$ perfect fourths from the 'top'. (This holds true even for chords 15-17, where the perfect fourths are inverted as perfect fifths. In these cases, the 'top' becomes the bottom.) Since the majority of the vocal pitch classes are chord notes, and nearly every chord contains at 
least one concurrent vocal pitch class, the series of relative ordinal positions forms a path that spans the entire song.

Example 2 displays one interpretation of this path. The vertical dimension of Example 2 represents the piano's harmonies as segments of the $\mathrm{T}_{5}$ cycle, with the sharpmost member of each - its lower limit - at the bottom, and the flatmost member of each - its upper limit - at the top. (The choice to stack the chords by fourths from bottom to top, thus orienting flat and sharp as up and down respectively, is motivated solely by the principal registral spacing used by the composer. From a strict pitch-class perspective, the vertical orientation could just as easily be inverted, suggesting that this dimension is better correlated with a more neutral 'left-to-right' than with a gravity-biased 'up-and-down'.) A horizontal alignment of the piano chords essentially takes the observer/listener 'inside' the cage/harmony, with the aim of making the composite motions more apparent. The boxed-in pitch classes are melodic chord notes in the vocal part: the solid boxes indicate the first chord note above each harmony, and the dotted boxes indicate the occasional second chord note. Within the graph of Example 2 is traced a left-to-right course, whose basic outline is marked with arrows that link together the first chord notes of the vocal line in each harmony. ${ }^{18}$ The composite trajectories from the two analytical vignettes considered earlier can be found along the path of Example 2: the linear stepwise movement during chords 1-3 is shown by a single rising arrow connecting chord notes $F \#, F \#$, and $D$ at the beginning of the graph, and the stasis within chords $8-10$ is indicated by a single arrow that runs parallel with the horizontal axis. Both these arrows signify what will be called successions - two or more adjacent motions in the composite space - whereas the term motion will be reserved for the change in relative position of a pitch class between adjacent chords. (In Example 2, the arrows connecting three or more pitch classes are successions, whereas those connecting two pitch classes are motions.)

This composite analysis is fairly objective - that is, given the parameters of the analytical method and the prioritization of first chord notes, anyone else would come up with the same path (or its inverse) - except in three places. The first two are chords 7 and 19, two registrally expanded structural pillars that highlight two punctuated breaks in the text. Example 3 lays out each chord on a circle of fourths, demonstrating that, from a pitch-class point of view, these two chords break from the pentatonic mould in two ways: not only do they have more than five pitch classes, but also their pitch classes cannot be arranged contiguously along the circle of fourths. But this fact does not necessarily exclude chords 7 and 19 outright from participating in the composite analysis. As shown by the enclosures in Example 3, each chord arranges at least half of its members into a contiguous segment of fourths. Lambert notes

18 Example 2 may remind some readers of a transformational voice-leading analysis akin to that first introduced in Klumpenhouwer, 'A Generalized Model'; in fact, it is a figure-ground reversal of such an analysis. In transformational voice leading, notes from consecutive chords are horizontally adjacent (the ground) if they belong to the same voice (soprano, alto, etc.) and notes from consecutive chords are connected by arrows (the figure) when they are related by the same transformation that relates the consecutive chords. In graphs such as Example 2, notes from consecutive chords are connected by arrows (the figure) when they belong to the voice part, and notes from consecutive chords are horizontally adjacent (the ground) when they are related by the same transformation that relates the consecutive chords. What permits a reversal for this analysis is that the members of the quartal pentachord may be totally ordered using perfect fourths just as the voice parts may be totally ordered in register. 

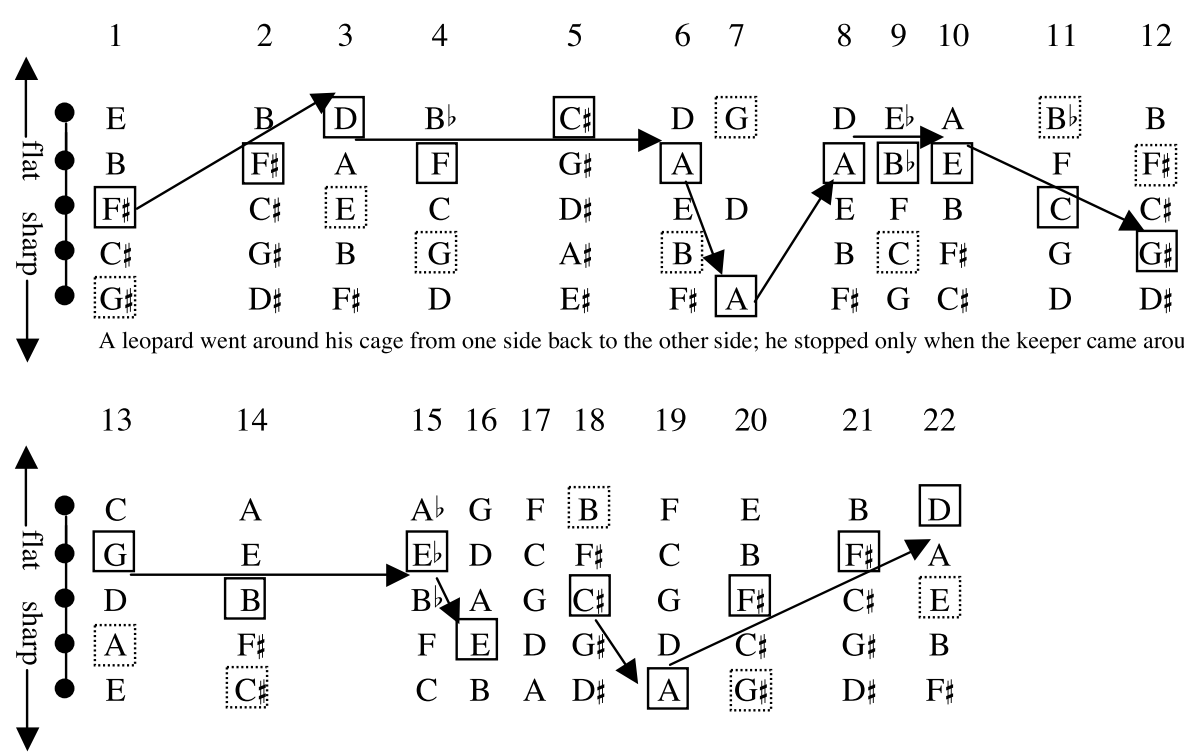

A boy who had been there three hours began to wonder, 'Is life anything like that?"

Example 2 Composite analysis of 'The Cage'. A solid-boxed pitch class is the first vocal chord note in the chord, and a dotted-boxed pitch class is the second vocal chord note in the chord (if any). Arrows indicate motions or successions.

that, although chord 7 'is more variously structured than the others, it does draw meaningful connections with the same cyclic source'. ${ }^{19}$ The most meaningful connection is the trichordal chain of perfect fourths A-D-G; this subset provides the best possible segment to represent chord 7. As shown in Example 2's vertical expansion of chord 7, this subset has been interpreted proportionally, in that, on account of its smaller size, it has been stretched so that its upper and lower limits align with those of the other segments. (In proportional segments, the unit of measure changes from ordinal to fractional: instead of being, for example, two perfect fourths from the top of the chord, a note is halfway from the top of the chord.) In the case of chord 19, the longest contiguous segment is the five-note subset A-D-G-C-F; likewise, this segment represents chord 19 in the graph of Example 2. ${ }^{20}$

The third anomaly is, as a quotation from Lambert noted earlier, that chord 17 is the only harmony in the song that does not support a chord note in the melody. However, this does not necessarily preclude the $\mathrm{E}$ from participating in the analysis. Since the $\mathrm{E}$ is a perfect fifth away from the chord note A, chord 17 can easily be extended sharpwards by one member to form the six-member segment $\mathrm{F}-\mathrm{C}-\mathrm{G}-\mathrm{D}-\mathrm{A}-\mathrm{E}$. In this segment $\mathrm{E}$ becomes the new sharpward limit, and chord 17 could be proportionally contracted as the segment representing chord 7 was proportionally expanded. Or the E could be conceived as lying just one step 'outside' chord 17, and the sharpward succession that led up to it could be interpreted as 'breaking through' the limits of the pentachordal segment. Either of these analyses seems viable. Yet since this analytical approach originated with the concept of overlap of pitch

19 Lambert, The Music of Charles Ives, 154.

20 Hitchcock's editorial choice of $\mathrm{D}^{3}$ and $\mathrm{F}^{3}$ over $\mathrm{D}^{3}$ and $\mathrm{F}^{3}$ (see note 1 ) is thus quite significant here. 
Chord 7

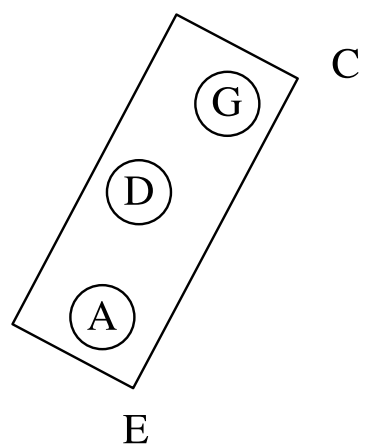

(B) $(\mathrm{FH}$

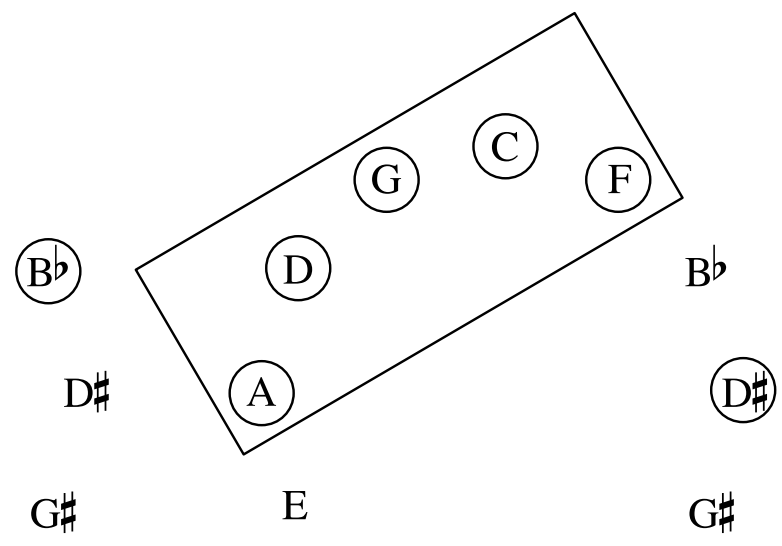

F

$\mathrm{G} \#$
Chord 19

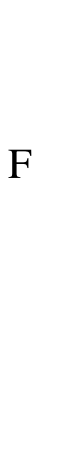

$\mathrm{C}$
(B)

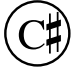

Example 3 Chords 7 and 19 situated on the pitch-class circle of fourths. The rectangular enclosures isolate the longest segment in each chord.

collections between piano and vocal parts, Example 2 excludes from its analysis any vocal note that is not doubled in the piano part.

What generalizations may be drawn from the path of Example 2? I believe the most significant generalization is the synchrony of each of the four punctuation marks in the text with either a single composite motion or a unidirectional succession of composite motions towards a limit of the perfect-fourth space. (The term 'unidirectional' describes a composite succession whose constituent motions are all either flatward or sharpward.) Each motion or succession spans at least two perfect-fourth steps in the composite space, and each ends either on a limit or one perfect-fourth step from a limit. Therefore the analysis will designate such single motions and successions as closed, for their size and placement in the composite space precludes a continuation of the motion or succession without breaching the well-established pentatonic limits of the perfect-fourth space. In other words, if both melody and harmony were to continue on their respective trajectories, trajectories that had commenced with the closed motion or succession, the melody would no longer be doubled by the harmony.

1. Preceding the semicolon of 'other side', the vocal line uses the A twice, but the harmonic shift from chord 6 to the representative segment of chord 7 suddenly shifts its location from a flatward position (where it has been lingering since chord 3 ) in the composite space to a sharpward limit. Along with the above-mentioned composite stasis accompanying the word 'stopped', this is the clearest bit of text painting in the composite space: the vocal line shifts to the space's 'other side' just as this text is enunciated.

2. The semicolon ${ }^{21}$ after 'meat' is preceded by a three-note closed succession from the flatward side to the sharpward side during chords 10-12. Not only is this composite 


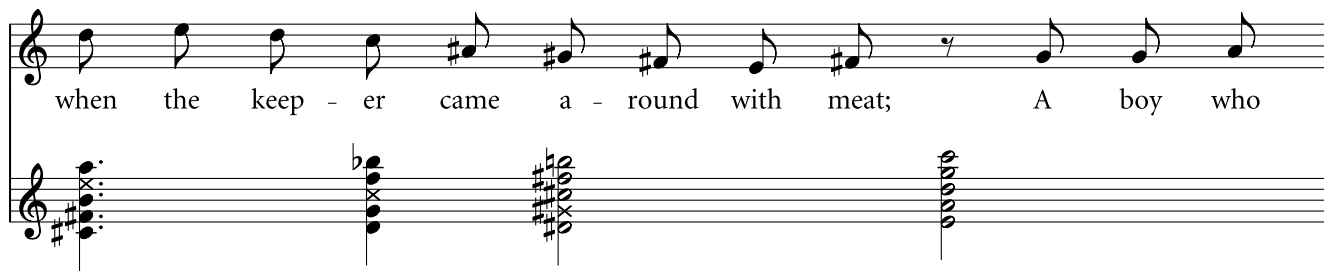

Example 4 Interaction in register between the vocal line and chords 10-12.

trajectory linear - answering with a sharpward direction the linear flatward succession during chords 1-3 - but its harmonic and melodic constituents are also moving on uniform courses in register: the harmony up by a semitone, and the melody down by a major third. Provided the melody is sung at notated pitch by a soprano or mezzo-soprano (instead of a tenor or baritone), it cuts transversely across both registral pitch and pitch-class harmonic space, as shown in Example 4, where the ' $X$ ' noteheads mark the points at which melody and harmony intersect. This registral relationship can help the listener perceive the oblique composite succession. ${ }^{22}$

3. The move from the vocal $\mathrm{C} \#$ in the middle of chord 18 to $\mathrm{A}$ on the sharpward edge of the representative segment of chord 19 ushers in the comma following 'wonder'. This twostep closed motion into chord 19 is not as large as the similar three-step closed motion into chord 7. Likewise, the punctuation is not as strong, nor the clause as closed: the verb 'wonder' could be either transitive or intransitive here, and could thus either precede an object ('A boy began to wonder if [...]') or conclude the sentence ('A boy began to wonder.').

4. Ives reuses the music of the opening text 'A leopard went around his cage' (chords 1-3) for the concluding text 'Is life anything like that?' (chords 20-22). Given the vocal part's relentless quavers, and the song's abrupt conclusion, which interrupts what was previously heard during chords $1-7$ as a unified section, there is good reason to analyse the song's close as a 'seemingly aborted statement'. ${ }^{23}$ Yet 'Is life anything like that?' is still a complete sentence, and its closure as signified by the question mark joins the culmination of a three-note closed succession from the middle to the flatward limit of the composite space. These two interpretations can coexist, relating to different aspects of the text. Furthermore, the end of the song presents the only four-note unidirectional succession in the compound space - in this case, from sharp to flat - and extends from one limit to the other. This interpretation requires that 'wonder' be included with the final question, which undeniably cuts across a more natural segmentation of the text. But it

22 The melodic and harmonic trajectories also anticipate an even stronger arrival at 'with': the melody predictably dropping to $\mathrm{E}^{4}$, and the harmony predictably and concurrently rising another semitone. But the melodic drop and the harmonic ascent do not coincide; rather the expected harmony is delayed a crotchet, accompanying new melodic material instead. This displacement thwarts a four-note unidirectional succession, of the kind that will eventually end the song.

23 Lambert, The Music of Charles Ives, 153. The truncated ending has been commented on by other scholars, including Starr, A Union of Diversities, 128: 'The verbal and musical unanswered question hangs in the shocked silence, lingering beyond the point where the piece stops - as Ives's fermata clearly indicates.' 
suggests one way in which the same passage of music can function differently as beginning and end.

Whereas each of the four punctuation marks corresponds to the end of a closed motion or succession, there are three closed motions or successions that do not coincide with punctuation. However, the termination of each coincides with an appropriate stopping point in the text and/or music. The closed succession of chords 1-3, along with the change of whole-tone scale, articulates the phrase 'A leopard went around his cage', which is Lambert's first division of the text. The closed motion of chords 7 and 8 coincides with the second change of whole-tone scale, and highlights 'stopped', which is allotted a dotted crotchet in the vocal part. Lambert in particular recognizes the option of interpreting the arrival at either chord 7 or chord 8 as a cadence; the two consecutive closed motions leave this option open as well. The closed motion of chords 15 and 16 underlines 'hours', to which Ives allots the only other dotted-crotchet duration in the entire vocal part.

While these generalizations hardly constitute a 'style' in the general sense of the word, they are consistent with other music of Ives that uses a very similar layering technique. Within Ives's published vocal output the pervasive use of pentatonic harmonies is unique to 'The Cage', yet a few other songs employ substantial stretches of quartal harmony. 'Majority', written fifteen years after the first version of 'The Cage', contains a passage whose piano accompaniment is reminiscent of the earlier song. ${ }^{24}$ Example 5 isolates the setting of the line 'The Masses are thinking, Whence comes the thought of the world!', and Example 6 provides a composite analysis of the passage, using the same criteria and notation introduced during analysis of the 'Cage'. Note that the quartal harmonies in this passage include a higher percentage of notes from the vocal line than those in 'The Cage': only the semiquavers $\mathrm{C} \#$ and $\mathrm{B} b$ are unequivocally non-chord notes, in that they are not simultaneously doubled in the piano part. ${ }^{25}$ Other non-doubled pitches can easily be analysed as displaced chord notes: the association of the first vocal note $\mathrm{E}$ with chord 3 (composed out by an F-E suspension in the voice) and the association of the peak vocal note F with chord 8 (composed out by a G-F suspension in the piano). Furthermore, unlike 'The Cage', 'Majority' contains only one instance of multiple chord notes for one harmony: the A and $\mathrm{E}$ within chord 6.

As in 'The Cage', all the harmonies in this excerpt from 'Majority' can be well represented as segments of the $\mathrm{T}_{5}$ cycle. However, unlike the 'The Cage', which uses five-note harmonic segments almost exclusively, the size of the harmonic collections in the passage from 'Majority' involves harmonic collections whose size varies frequently and markedly: the piano chords express two four-note segments, five five-note segments, three six-note segments, and two seven-note segments. As with chord 7 in 'The Cage', the composite analysis

24 It is perhaps no coincidence that, for the one verse in 'Majority' where the masses are at their most pensive, Ives uses a harmonic style that harks back to a song with one of his most philosophical texts.

25 The vocal note $\mathrm{B} b$ is not a member of chord 4, though it is adjacent to the flatward $\mathrm{F}$ along the circle of perfect fourths; therefore, as with the E above chord 17 in 'The Cage', it has not been included in the analysis, since until now I have designated as chord notes only those pitches doubled in the accompaniment. However, this is certainly not the only way to consider such vocal notes that are just 'outside' their concomitant harmony. 

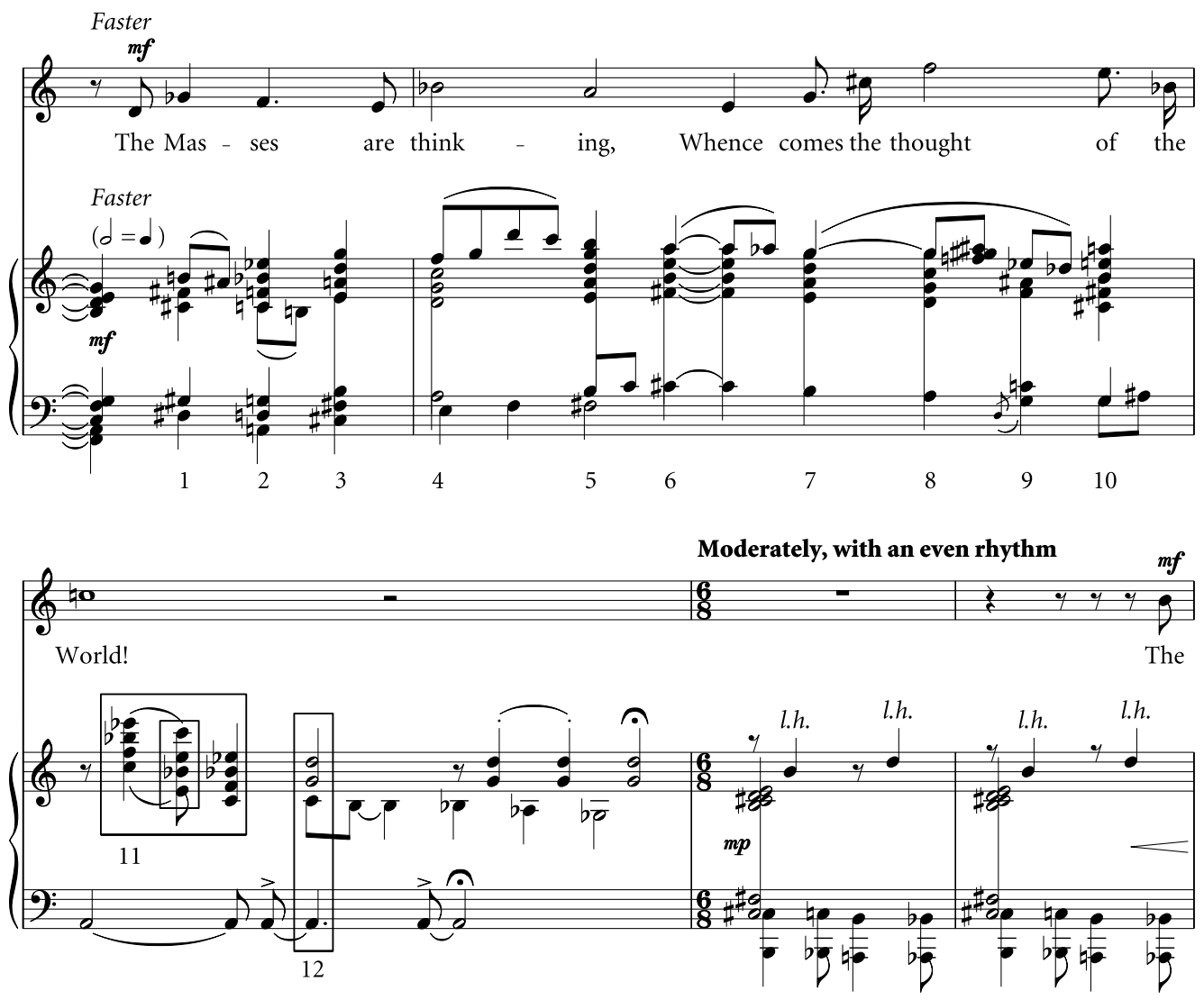

Example 5 Ives, 'Majority’ @ 1935, Merion Music Inc., Bryn Mawr, PA 19010. Theodore Presser Co., sole representative. International Copyright Secured. Reproduced by kind permission of Theodore Presser Co. / United Music Publishers Ltd. Bars 17-21, with numerical labelling of chordal events. Accidentals apply only to notes that they immediately precede.

accommodates this variety through a proportional consideration of these segments, as shown in Example 6, where the upper and lower limits of all the segments are aligned.

As in 'The Cage', a closed composite motion or succession accompanies each of the two instances of textual punctuation. But, unlike in 'The Cage', this text-music correspondence is also one-to-one, as the following brief tour of the passage will illustrate. The vocal trajectory over the course of chords 1 to 3 projects a gradual sharpward succession from near the periphery of the quartal segment to its centre: here, a non-closed succession accompanies the beginning of a clause. As an aside, this net stepwise succession is the difference between the semitonal descent in the voice and the accompanying major-third ascent in the piano chords, measured from the flatward limits of $\mathrm{B}, \mathrm{Eb}$, and $\mathrm{G}$. This is precisely the inverse of the way in which Ives achieves net stepwise succession in chords 10 to 12 in 'The Cage': the voice drops by major thirds - fleshed out by a whole-tone scale - while the piano chords ascend by semitone.

Local extremes colour the arrival at chord 4 with the upward leap to the non-chord note $\mathrm{B} b$ in the voice and the minims in both voice and piano parts; Ives's barline visually affirms these 


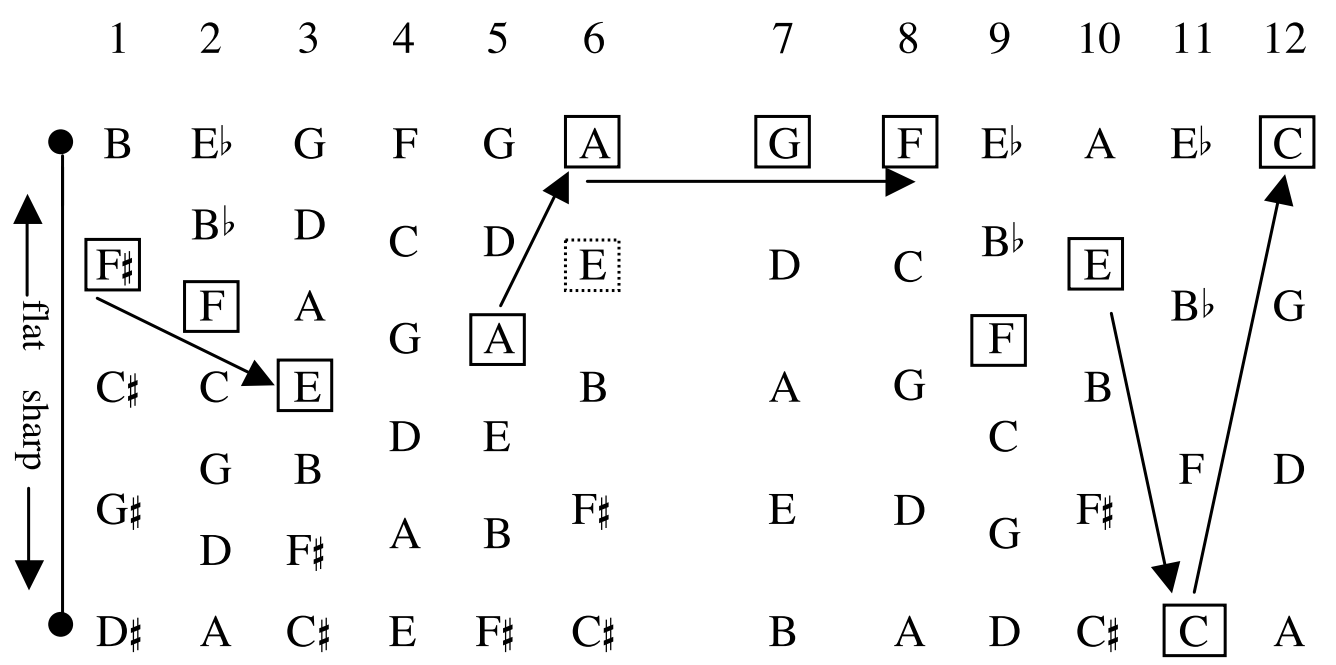

The mas- ses are think- ing, Whence comes the thought

of the world!

Example 6 Composite analysis of bars 17-19 of 'Majority'.

emphases. The textual punctuation after 'thinking' is coupled with a closed motion, as the vocal A is sustained above chords 5 and 6. (To hear this closed composite motion, it is important that the singer sustain the A into chord 6 for at least a quaver, preferably accompanied by a slight ritardando.) Following this closed motion, both the first three melodic chord notes, A, G, and F, and chords 6-8 'descend' twice by a major second; this parallelism becomes a static succession within the compound segment. Again, this non-closed succession begins the new textual phrase 'Whence comes the thought of the World!'.

The conclusion of this phrase boasts two adjacent closed motions, each of which is larger than the preceding closed motion. The first motion, from the $\mathrm{E}$ in chord 10 to the $\mathrm{C}$ in chord 11 , accompanies the arrival at 'World!', which is also affirmed by the semibreve duration of the vocal note and the relatively low bass note $\mathrm{A}^{2}$. This motion spans nearly the entire composite space. However, the motion from chord 11 to chord 12 about the common tone $\mathrm{C}$ is, like the final succession in 'The Cage', the only motion to proceed from one limit of the segment to the other, providing maximal closure. Again, if this final composite motion is to be perceived, the singer should sustain the vocal note $C$ to its full length, which is long enough for this concluding note to coincide with chord 12 .

In his article on spatial form in Ives Robert P. Morgan does not discuss 'The Cage' in particular, but he considers the general category of pieces and excerpts from pieces in which Ives presents contrasting musical ideas simultaneously:

The most essential point [...] is that such passages are textually, and thus temporally, multidimensional. The absence of a single strand of continuity - what Roger Sessions used to refer to as 'the long line' - reflects a basic shift in compositional 
orientation. Ives works with a number of different yet simultaneous timemovements coexisting in a shared, multilayered universe where each maintains its own individuality while also influencing and being influenced by all others. ${ }^{26}$

Morgan's pluralist stance calls for a balance of analytical approaches to Ives's layered compositions between, on the one hand, those that focus on each layer separately and, on the other hand, those that focus on the intersections between and among layers. In 'The Cage', although many factors suggest to music analysts that each of the two parts should 'maintain its own individuality', there is a potential perceptual 'influence' between these two parts, where a listener's interpretation of a vocal note may be coloured by the context provided by its supporting piano harmony. The composite analysis outlined above offers one method of interpreting this influence, but certainly not the only method for analysing either this piece in particular or, especially, other layered pieces in general, given the many different kinds of layering that Ives explored. And although my method may be somewhat removed from the way in which Starr originally conceived of a 'composite style' in 'The Cage', I take comfort in his conviction that, in the case of Ives, iconoclastic music that employs a heterogeneity of styles invites a heterogeneity of traditional and new analytical approaches; ${ }^{27}$ it is to the author of this conviction that this analysis is dedicated.

\section{Bibliography}

Carr, Cassandra I. 'Charles Ives's Humor as Reflected in His Songs'. American Music 7/2 (1989), 123-39.

Euteneuer-Rohrer, Ursula Henrietta. 'Charles E. Ives: The Cage'. Neuland 1 (1980), 47-51.

Hitchcock, H. Wiley. 'Editing Ives's 129 Songs', in Ives Studies, ed. Philip Lambert. Cambridge: Cambridge University Press, 1997. 51-76.

Ives, Charles. 129 Songs, ed. H. Wiley Hitchcock. Middleton, WI: A-R Editions, 2004.

Kingman, Daniel. American Music: a Panorama, 2nd edn. New York: Schirmer, 1990.

Klumpenhouwer, Henry. 'A Generalized Model for Voice-Leading in Atonal Music'. PhD diss., Harvard University, 1991.

Lambert, Philip. 'Ives and Berg: "Normative" Procedures and Post-Tonal Alternatives', in Charles Ives and the Classical Tradition, ed. Geoffrey Block and J. Peter Burkholder. New Haven: Yale University Press, 1996. 105-30.

- The Music of Charles Ives. New Haven: Yale University Press, 1997.

Lewin, David. Generalized Musical Intervals and Transformations. New Haven: Yale University Press, 1987. 83-120.

Morgan, Robert P. 'Spatial Form in Ives', in An Ives Celebration, ed. H. Wiley Hitchcock and Vivian Perlis. Urbana: University of Illinois Press, 1977. 145-58.

Nicholls, David. American Experimental Music, 1890-1940. Cambridge: Cambridge University Press, 1990.

O’Donnell, Shaugn J. 'Transformational Voice Leading in Atonal Music'. PhD diss., City University of New York, 1997.

Starr, Larry. A Union of Diversities: Style in the Music of Charles Ives. New York: Schirmer, 1992.

26 Morgan, 'Spatial Form in Ives', 153.

27 Starr, A Union of Diversities, 30-32. 
Reproduced with permission of the copyright owner. Further reproduction prohibited without permission. 